\title{
Gender-dependent effect of Gpbar1 genetic deletion on the metabolic profiles of diet-induced obese mice
}

\author{
Galya Vassileva, Weiwen Hu, Lizbeth Hoos ${ }^{1}$, Glen Tetzloff ${ }^{1}$, Shijun Yang, Li Liu ${ }^{1}$, Ling Kang ${ }^{1}$, \\ Harry R Davis ${ }^{1}$, Joseph A Hedrick ${ }^{1}$, Hong Lan ${ }^{1}$, Timothy Kowalski ${ }^{1}$ and Eric L Gustafson \\ Merck Research Laboratories, Department of Discovery Technologies, Kenilworth, New Jersey 07033, USA \\ ${ }^{1}$ Merck Research Laboratories, Department of Cardiovascular and Metabolic Diseases, Kenilworth, New Jersey 07033, USA \\ (Correspondence should be addressed to G Vassileva; Email: galya.vassileva@spcorp.com)
}

\begin{abstract}
G-protein-coupled bile acid receptor 1 (GPBAR1/TGR5/ M-Bar/GPR131) is a cell surface receptor involved in the regulation of bile acid metabolism. We have previously shown that Gpbar1-null mice are resistant to cholesterol gallstone disease when fed a lithogenic diet. Other published studies have suggested that Gpbar1 is involved in both energy homeostasis and glucose homeostasis. Here, we examine the functional role of Gpbar1 in diet-induced obese mice. We found that body weight, food intake, and fasted blood glucose levels were similar between Gpbar1-null mice and their wild-type (WT) littermates when fed a chow or high-fat diet (HFD) for 2 months. However, insulin tolerance tests
\end{abstract}

revealed improved insulin sensitivity in male Gpbar1 $1^{-/-}$ mice fed chow, but impaired insulin sensitivity when fed a HFD. In contrast, female Gpbar $1^{-/-}$mice exhibited improved insulin sensitivity when fed a HFD compared with their WT littermates. Female Gpbar1 $1^{-1-}$ mice had significantly lower plasma cholesterol and triglyceride levels than their WT littermates on both diets. Male Gpbar1 $1^{-/-}$ mice on HFD displayed increased hepatic steatosis when compared with Gpbar1 $1^{+/+}$males and Gpbar $1^{-/-}$females on HFD. These results suggest a gender-dependent regulation of Gpbar1 function in metabolic disease.

Journal of Endocrinology (2010) 205, 225-232

\section{Introduction}

G-protein-coupled bile acid receptor 1 (GPBAR1), also known as TGR5 (Kawamata et al. 2003) and M-BAR (Maruyama et al. 2002), is the founding member of the bile acid (BA) receptor subclass of G-protein-coupled receptors (Foord et al. 2005). GPBAR1 is a $\mathrm{G}_{\mathrm{s}}$-coupled cell surface receptor that is expressed on the plasma membrane, and is internalized in response to agonist activation (Kawamata et al. 2003). BA-induced cellular effects of GPBAR1 activation include stimulation of cAMP synthesis (Houten et al. 2006), and have been implicated in a range of cellular and physiological activities (Thomas et al. 2008a,b, Fiorucci et al. 2009, Wei et al. 2009).

GPBAR1 mRNA has been detected in a variety of tissues in human, rats, and mice. In mice, it is predominantly expressed in the gallbladder (Vassileva et al. 2006), but also in gastrointestinal tract, lung, heart, ovary, placenta, white adipose tissue (WAT), and brown adipose tissue (BAT; Maruyama et al. 2002, 2006, Kawamata et al. 2003, Vassileva et al. 2006, Watanabe et al. 2006). In mouse gallbladder, the expression level of Gpbar1 mRNA is 60-100 times greater than that of Gpbar1 mRNA in all other tissues analyzed, and in situ hybridization has shown that it is restricted to epithelial cells (Vassileva et al. 2006). Recently, GPBAR1 protein was localized to liver sinusoidal endothelial cells and Kupffer cells in rat (Keitel et al. 2007, 2008a), and gallbladder epithelium in human (Keitel et al. 2009). GPBAR1 is expressed in enteroendocrine cell lines, such as NCI-H716, STC-1, and GLUTag (Maruyama et al. 2002), and has been shown to stimulate the production of glucagon-like peptide 1 (GLP-1) in STC-1 cells (Katsuma et al. 2005).

BAs are known regulators of energy homeostasis and glucose metabolism that have been reported to inhibit dietinduced obesity and prevent the development of insulin resistance (Ikemoto et al. 1997, Watanabe et al. 2006, Kobayashi et al. 2007, Keitel et al. 2008b). Watanabe et al. (2006) have shown that administration of BAs to mice increases energy expenditure in BAT, preventing obesity and insulin resistance. This is achieved via the induction of the cAMP-dependent thyroid hormone activating enzyme type 2 iodothyronine deiodinase (Dio2). Treatment of mouse brown adipocytes and human skeletal myocytes by BAs increases Dio2 activity and oxygen consumption. These effects are independent of FXR and are mediated by increased cAMP production, resulting from GPBAR 1 activation by BAs. Since mouse BAT and human skeletal muscle co-express Dio2 and GPBAR1, it has been suggested that GPBAR1 regulates energy expenditure. GLP-1 secretion has been found to be induced by BAs through GPBAR 1 in a murine 
enteroendocrine cell line STC-1 (Katsuma et al. 2005). Oleanolic acid, a natural GPBAR1 agonist isolated from olive leaves (Olea europaea), decreased plasma glucose and insulin levels when administered to diet-induced obese C57BL/6 mice (Sato et al. 2007). These findings suggest a role of GPBAR1 in glucose homeostasis. Recently, Thomas et al. (2009) showed that activation of GPBAR1/TGR 5 by a selective agonist INT-777 results in an increase in intracellular levels of cAMP, oxygen consumption, cytochrome $c$ oxidase, and the ATP:ADP ratio, leading to an improved liver and pancreatic function and enhanced glucose tolerance in obese mice.

We have previously shown that Gpbar1 plays a critical role in the formation of gallstones in mice (Vassileva et al. 2006), and that the mice with targeted deletion of this gene are protected from gallstone disease. Maruyama et al. (2006) have reported that independently generated knockout $(\mathrm{KO})$ mice have smaller total $\mathrm{BA}$ pool size, and that female $\mathrm{KO}$ mice accumulate fat and gain body weight (BW) when placed on high-fat diet (HFD) for 2 months. In this study, we investigated the role of Gpbar1 in diet-induced obese mice, and found that male and female Gpbar $1^{-1-}$ mice respond differently to HFD challenge. There were differences between the two sexes in their plasma lipoprotein profile, response to insulin tolerance test (ITT), and liver function. These results suggest a gender-dependent regulation of Gpbar1 function.

\section{Materials and Methods}

\section{Animals and diets}

Gpbar1 KO mice in C57BL/6J background were generated as described in Vassileva et al. (2006) (Supplementary information). Mice were bred in house, and age- and gendermatched animals were used between 8 and 20 weeks of age. All mice were housed in a specific pathogen-free environment, and all experiments with animals were conducted according to the Schering-Plough ACUC guidelines for animal care.

Diets were prepared by Research Diets, Inc. (New Brunswick, NJ, USA). Mice were maintained on normal chow, containing $<0.02 \%$ cholesterol, until 8 weeks of age. Wild-type (WT) and $\mathrm{KO}$ littermates were then fed a HFD (D12492) containing $60 \mathrm{kcal} \%$ fat. BWs were measured weekly. After feeding the HFD for 9 weeks, mice were killed, and their tissues were collected for further analyses.

\section{Plasma and hepatic lipid measurements}

Plasma samples were stored at $4{ }^{\circ} \mathrm{C}$ and analyzed by fast protein liquid chromatography (FPLC) to separate lipoproteins by gel permeation chromatography. The FPLC system, AKTA Basic (GE Healthcare, Piscataway, NJ, USA), comprised the following components: P-920 pumps, INV907 and PV-908 valves, M-925 mixer, UPC-900 monitor, 100- $\mu$ l sample loop, Frac-950 fraction collector, Superose 6 100/300 GL Tricorn column (C/N 0827026), and Unicorn operating system. Plasma samples manually loaded into the $100-\mu$ loop were eluted with a degassed buffer containing DPBS without calcium and magnesium (Gibco C/N 14190) with added $10 \mathrm{ml} / 1$ of $5 \mathrm{M}$ sodium chloride (Sigma, S-5150), $2 \mathrm{ml} / 1$ of $0.5 \mathrm{M}$ disodium EDTA (Sigma, E-7889), and $5 \mathrm{ml} / 1$ of $4 \%$ sodium azide (Sigma, S8032) at a rate of $0.5 \mathrm{ml} / \mathrm{min}$. After a column equilibration period of $10 \mathrm{~min}$, the samples were injected. Subsequently, 40 0.5-ml fractions were collected beginning at $9 \mathrm{~min}$ after injection. Cholesterol content in each fraction was determined by a modification of the cholesterol oxidase method of Allain et al. (1974) using Wako test kits (Wako Chemicals, Richmond, VA, USA): cholesterol E (C/N 439-17501). Triglyceride (TG) concentrations were determined using the Wako L-Type TG H reagents (C/N 997-37492 and 993-37592). Forty microliters from each fraction or standards were mixed with $200 \mu$ l of the appropriate reagent and incubated in an oven at $40{ }^{\circ} \mathrm{C}$ for $15 \mathrm{~min}$, and absorbance was determined in a Molecular Devices plate reader at $600 \mathrm{~nm}$. Results were calculated from standard curves, and reported as micrograms of cholesterol or TGs per fraction. Quantities from fractions 15-19, 20-28, and 29-36 were summated to be reported as $\mathrm{mg} / \mathrm{dl}$ of cholesterol or TGs in very low-density lipoprotein (VLDL), low-density lipoprotein (LDL), and high-density lipoprotein (HDL) respectively.

Liver samples $(\sim 250 \mathrm{mg})$ were frozen and stored at $-20{ }^{\circ} \mathrm{C}$ until analysis. Individual samples were extracted according to the procedure of Folch et al. (1957) and homogenized in $6 \mathrm{ml}$ of chloroform:methanol (2:1), and $4 \mathrm{ml}$ of water were added, vortexed, and centrifuged at $1000 \mathrm{~g}$ for $30 \mathrm{~min}$. The chloroform layer was removed and dried under nitrogen. The extracted lipids were redissolved in $1 \mathrm{ml}$ chloroform, and $0.1 \mathrm{ml}$ was transferred into HPLC sample vials. Samples were dried under nitrogen and redissolved in $1 \mathrm{ml}$ of hexane:isopropanol $(98 \cdot 8: 1 \cdot 2)$. Chromatography was performed essentially as described by Burrier et al. (1995) using an isocratic mobile phase containing $98.8 \%$ hexane and $1 \cdot 2 \%$ isopropanol at a flow rate of $2 \mathrm{ml} / \mathrm{min}$ through a Zorbax Sil $(4.6 \times 25 \mathrm{~cm})$ silica column (Agilent Technologies \# 880952-701, Parsippany, NJ, USA). Lipids in a 5- $\mu 1$ injection were detected by absorbance at $206 \mathrm{~nm}$ and quantitated by computer integration (System Gold, Beckman, Fullerton, CA, USA) of AUCs. Cholesterol, cholesteryl ester, and TG concentrations were determined by comparison to standard curves using Non-polar Lipid Mix-B, Matreya, Inc., Pleasant Gap, PA, USA, C/N 1130. The accumulation of hepatic free cholesterol (FC) and cholesteryl esters was used as a surrogate marker of cumulative cholesterol absorption and its inhibition in mice (Salisbury et al. 1995). Data were reported as $\mathrm{mg} / \mathrm{g}$ liver and $\mathrm{mg} /$ liver.

\section{Blood glucose analysis}

For blood glucose levels, Gpbar1 $1^{-/-}$and Gpbar1 $1^{+/+}$ littermate mice were fasted for $4 \mathrm{~h}$. Blood was collected from the tail, and glucose was measured using glucose oxidase 
method (Glucometer Elite, Bayer). For ITT, tail blood was collected after $5 \mathrm{~h}$ of fasting, and basal glucose levels were measured using a glucose oxidase method (Glucometer Elite, Bayer). After this measure, insulin $(0.75 \mathrm{U} / \mathrm{kg} \mathrm{BW})$ was administered by i.p. injection, and blood was collected from the tail vein at 20, 40, 60, 90, and $120 \mathrm{~min}$ after the administration of dose for glucose determination.

\section{Histological analysis}

Mice were killed, and their livers were collected, immersion fixed in $10 \%$ neutral-buffered formalin, and paraffin embedded, and sections were stained by standard hematoxylin and eosin (H\&E) methods. All tissues were examined by light microscopy and graded for severity by one investigator.

\section{Pancreatic islets isolation}

Pancreatic islets were isolated by collagenase digestion and discontinuous Ficoll gradient separation, a modification of the original method of Li et al. (2003). The islets were maintained in RPMI 1640 medium (11 mmol/l glucose, Invitrogen). The culture medium was supplemented with $10 \%$ fetal bovine serum, 100 units $/ \mathrm{ml}$ penicillin, and $100 \mu \mathrm{g} / \mathrm{ml}$ streptomycin, and islets were incubated at $37^{\circ} \mathrm{C}$ in a $5 \%$ $\mathrm{CO}_{2} / 95 \%$ air-humidified incubator.

\section{$m R N A$ expression analysis}

mRNA from various tissues was extracted utilizing the Ultraspec RNA isolation kit from Biotecx (Houston, TX, USA) following specifications from the manufacturer. cDNA was generated by reverse transcription using random hexamers (Promega) and oligo-dT primers (Life Technologies). Quantitative real-time PCR analysis was performed on an ABI 7900 sequence-detection instrument (TaqMan) following the manufacturer's instructions. Primers sequences for the genes involved in the cholesterol, BA, fatty acid, and glucose metabolism are shown in Supplementary Table 1, see section on supplementary data given at the end of this article. Ribosomal RNA primers (PE Applied Biosystems, Foster City, CA, USA) were used as an internal control. Quantitative PCR conditions were as follows: $50{ }^{\circ} \mathrm{C}$ for $2 \mathrm{~min} ; 95^{\circ} \mathrm{C}$ for $10 \mathrm{~min} ; 40$ cycles of $95^{\circ} \mathrm{C}$ for $15 \mathrm{~s}, 60^{\circ} \mathrm{C}$ for $1 \mathrm{~min}$. Data were analyzed using Sequence Detection Systems software version 1.7.

\section{Statistical analysis}

GraphPad Prism version 4 was used for graphics (GraphPad Software, San Diego, CA, USA). Values are expressed as means \pm S.E.M. Statistical comparisons for significance between groups were performed using Student's $t$-test. A probability value of $P<0.05$ was considered significant.

\section{Results}

Gpbar1 ${ }^{-/-}$mice fed HFD were indistinguishable by weight from their WT littermates

Gpbar1 $1^{-1-}$ males $(n=15)$ and females $(n=17)$ and Gpbar $^{+/+}$littermate males $(n=12)$ and females $(n=11)$ were fed a HFD for 8 weeks. Similar number of Gpbar ${ }^{-1-}$ and Gpbar1 ${ }^{+/+}$control mice were fed chow. There was no difference in the food consumption between the groups (data not shown). The BWs of both male and female Gpbar1 ${ }^{-1-}$ mice on chow were similar to those of their WT littermates (Fig. 1). As expected, both male and female Gpbar $1^{+/+}$mice on HFD became significantly heavier than the male and female Gpbar $1^{+/+}$control mice on chow. Gpbar1 ${ }^{-/-}$males and females on HFD also gained significantly more BW than the control Gpbar1 $1^{-1-}$ male and female mice on chow. The BWs of male and female Gpbar1 $1^{-/-}$mice were not statistically different from those of their WT littermates throughout the study, both on chow and on HFD, although the Gpbar1 $1^{-/-}$males on HFD were slightly heavier than the Gpbar $1^{+/+}$control males on HFD (Fig. 1).

\section{Gpbar1-/- females have lower plasma lipoprotein levels}

Cholesterol and TG levels in hepatic samples were measured at the end of the HFD study (Fig. 2). Gpbar $1^{-1-}$ males on HFD had lower FC, and Gpbar1 $1^{-1-}$ females on chow had elevated FC levels compared with their control groups. Plasma cholesterol levels in VLDL (Chol-VLDL), LDL (Chol-LDL), and HDL (Chol-HDL) fractions, as well as the total plasma cholesterol, were significantly lower in Gpbar1 ${ }^{-/-}$females on both chow and HFD compared with their WT littermate controls (Fig. 3A). Male Gpbar1 ${ }^{-/-}$ mice on chow showed lower total plasma cholesterol and Chol-LDL (Fig. 3A). Total plasma TG levels and TG levels in VLDL (TG-VLDL), LDL (TG-LDL), and HDL (TG-HDL)

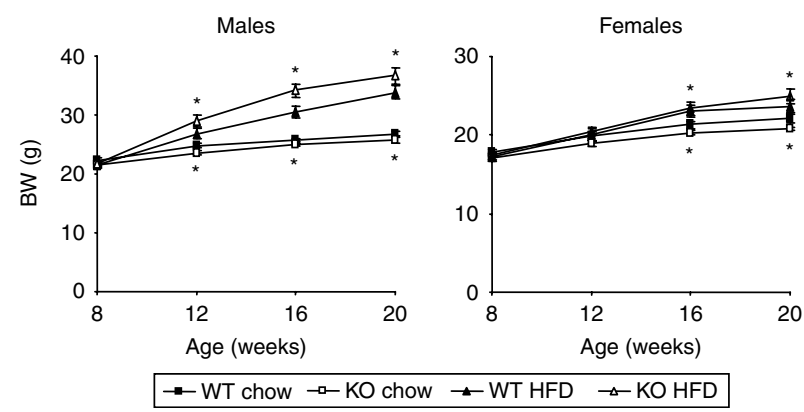

Figure 1 Body weights of Gpbar1 $1^{-1-}$ and Gpbar1 $1^{+/+}$mice. Individual values were measured from 11 to 17 mice per group on chow and HFD. Significantly higher body weights at 12, 16, and 20 weeks of age for WT males on HFD versus WT males on chow, and $\mathrm{KO}$ males on HFD versus $\mathrm{KO}$ males on chow. At 16 and 20 weeks of age, female WT mice on HFD show significantly higher BW than WT females on chow, and female KO mice on HFD are significantly heavier than the female $\mathrm{KO}$ mice on chow. Error bars represent \pm S.E.M. $* P<0 \cdot 05$. 

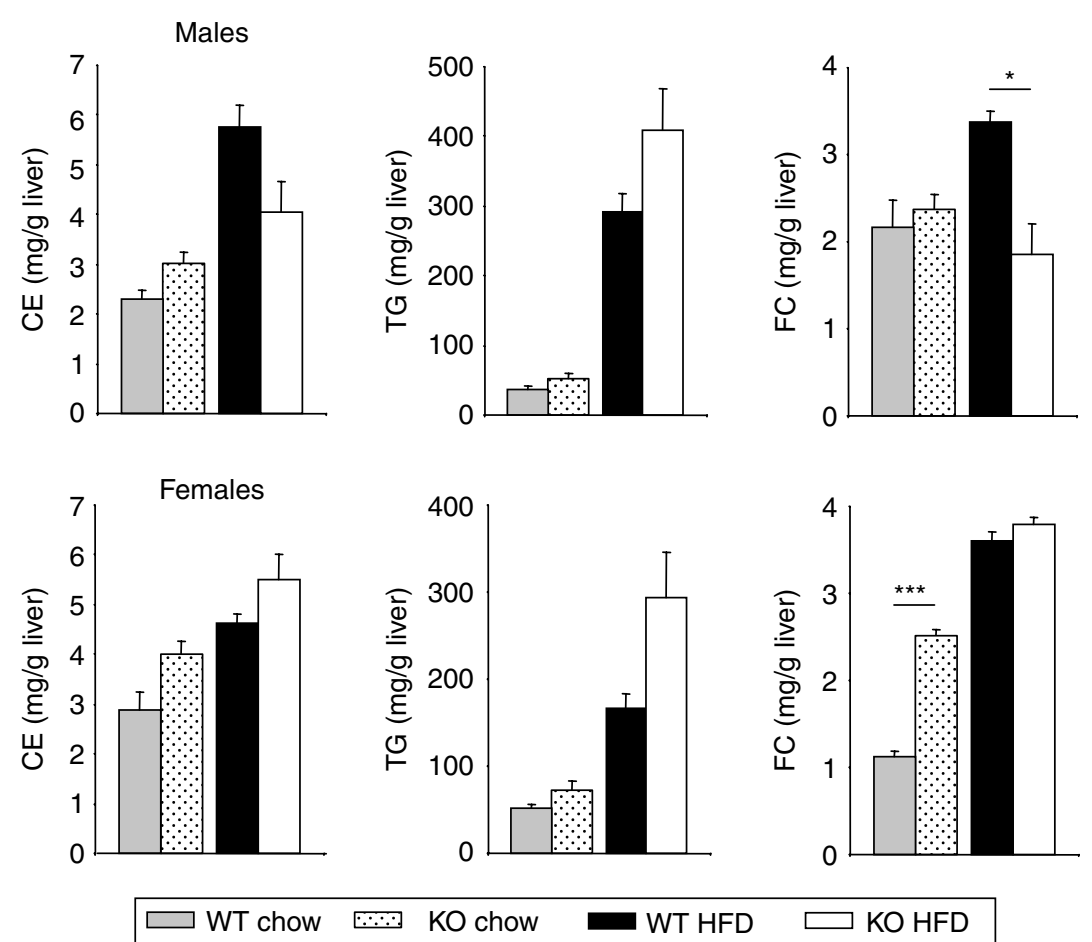

Figure 2 Hepatic lipid profile of Gpbar1 ${ }^{-1-}$ and $G p b a r 1^{+/+}$mice. Individual values were measured from 11 to 17 mice per group on chow and HFD. Error bars represent \pm s.E.M. ${ }^{*} P<0 \cdot 05$, and ${ }^{* * *} P<0 \cdot 001$. CE, cholesteryl esters; TG, triglycerides; FC, free cholesterol.

fractions were again significantly lower in Gpbar1 ${ }^{-/-}$ females on chow, but only TG-VLDL levels were lower in Gpbar1 ${ }^{-1-}$ females on HFD (Fig. 3B). In contrast, Gpbar $^{-/-}$males on both diets had TG levels comparable to those in their WT littermates with the exception of lower total TG and TG-LDL levels (Fig. 3B).

Gpbar1 $1^{-1-}$ males have improved insulin sensitivity on chow diet but impaired sensitivity on HFD

Blood glucose levels of Gpbar1 ${ }^{-1-}$ mice on chow were similar to those in their WT littermates at 8 and 16 weeks of age (Fig. 4A). As expected, WT mice on HFD became hyperglycemic after 8 weeks on HFD (Fig. 4A). Male Gpbar $^{-1-}$ on HFD had similar blood glucose levels to those in their WT littermates. Female Gpbar1 ${ }^{-/-}$mice on HFD had lower, but not significant, glucose levels than their WT littermates.

The ITT revealed improved insulin sensitivity in male Gpbar1 ${ }^{-/-}$mice fed chow at all time points the blood glucose was measured after insulin administration. But when fed HFD, male Gpbar1 ${ }^{-/-}$mice showed impaired insulin sensitivity at 30 and $60 \mathrm{~min}$ after insulin administration (Fig. 4B). Female Gpbar1 ${ }^{-1-}$ mice on chow were not different from their WT littermates, but when fed HFD they exhibited improved insulin sensitivity 60, 90, and $120 \mathrm{~min}$ after insulin administration (Fig. 4B).
Increased liver steatosis in Gpbar1 ${ }^{-/-}$males on HFD

After 8 weeks on HFD, Gpbar1 $1^{+/+}$and Gpbar1 ${ }^{-/-}$male and female mice were killed, and their livers were collected for histological analysis. As expected (Hoffler et al. 2009), Gpbar ${ }^{+/+}$males and females fed HFD developed hepatic lipidosis (steatosis) (Fig. 5A and C). The steatosis was in the form of microvesicular vacuolation of hepatocyte cytoplasm, characterized by small, round, clear vacuoles within the cytoplasm of hepatocytes, and consistent with the accumulation of lipids. Livers from female Gpbar $1^{-/-}$mice on HFD were comparable to those from the Gpbar $1^{+/+}$littermates displaying microvesicular steatosis (Fig. 5C and D). However, when compared with their WT littermates, Gpbar1 ${ }^{-1-}$ males showed an elevated hepatic lipid accumulation (Fig. 5A and B). Their hepatocytes contained large, round, clear vacuoles (macrovesicular vacuolation).

Expression of genes involved in cholesterol, BA, fatty acid, and glucose metabolism in Gpbar1 ${ }^{-1-}$ mice on HFD

In order to investigate the molecular basis of the response of Gpbar1 $1^{-/-}$mice to HFD challenge, we performed real-time quantitative PCR analysis of mRNAs isolated from pancreatic islets, BAT, WAT, and livers of Gpbar1 $1^{-/-}$ and Gpbar $1^{+/+}$mice fed HFD $(n=3)$. First, we selected 11 genes involved in the glucose homeostasis, and analyzed 

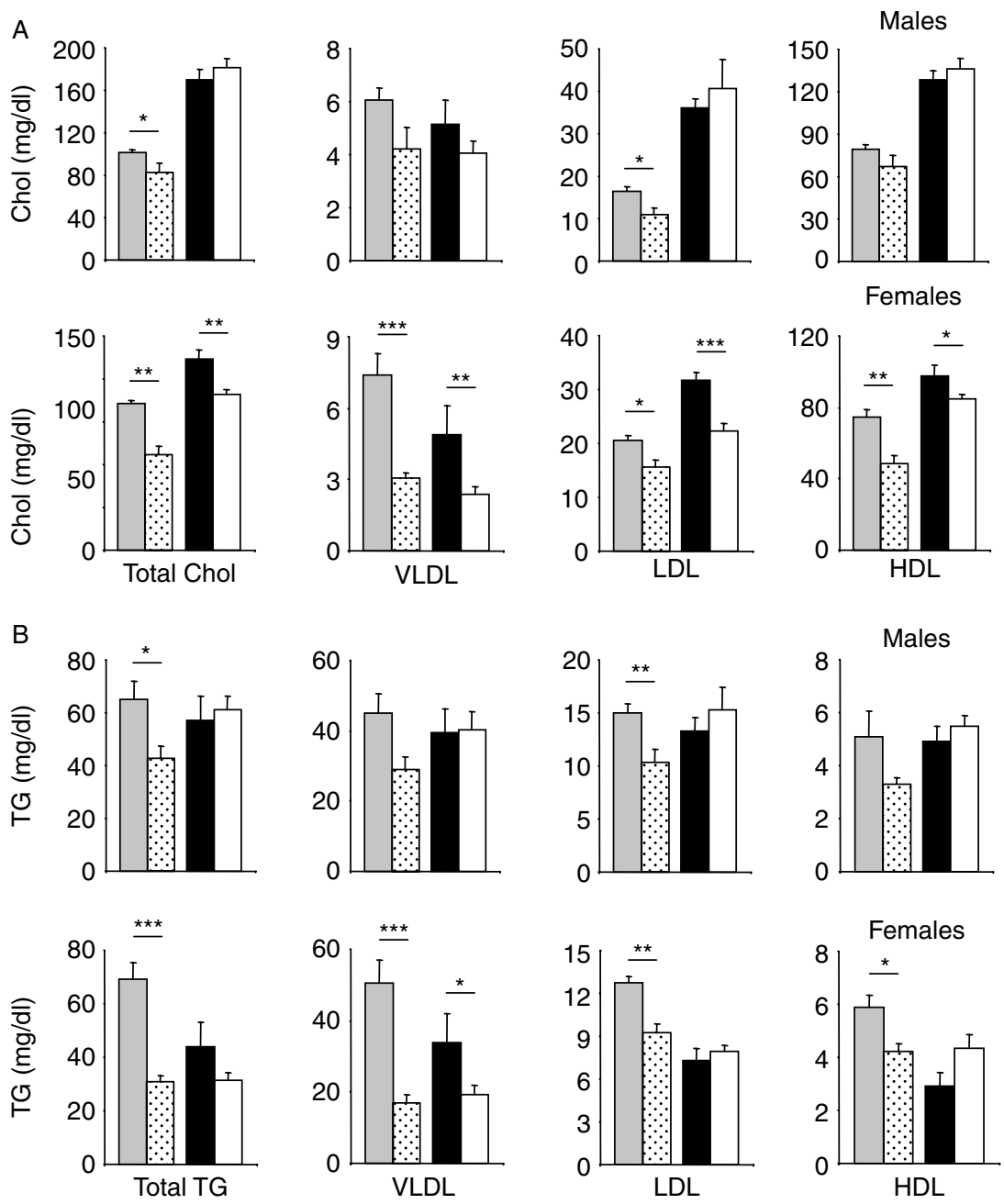

$\square$ WT chow $\because \because]$ KO chow

Figure 3 Plasma lipid profile of $G p b a r 1^{-1-}$ and Gpbar1 ${ }^{+/+}$mice: (A) cholesterol and (B) triglycerides. Individual values were measured from 11 to 17 mice per group on chow and HFD. Error bars represent \pm S.E.M. ${ }^{*} P<0 \cdot 05,{ }^{* *} P<0 \cdot 01$, and ${ }^{* * *} P<0 \cdot 001$. Chol, cholesterol; TG, triglycerides; VLDL, very low-density lipoprotein; LDL, low-density lipoprotein; HDL, high-density lipoprotein.

their mRNA expression levels in pancreatic islets. Glucagon levels were elevated twofold in male Gpbar1 ${ }^{-1-}$ mice on HFD when compared with Gpbar $1^{+/+}$littermates, and female Gpbar1 ${ }^{-1-}$ mice had a slightly decreased glucagon receptor (Gcgr) levels when compared with Gpbar $1^{+/+}$littermates on HFD (Supplementary Figure 1A, see section on supplementary data given at the end of this article).

We then studied the mRNA expression levels of 43 genes involved in cholesterol, BA, fatty acid, and glucose metabolism in BAT, WAT, and livers of Gpbar $1^{-1-}$ and $\mathrm{Gpbar}^{+/+}$mice fed HFD. In BAT and WAT, the mRNA levels of several genes were either elevated or decreased in male Gpbar $1^{-1-}$ mice compared with Gpbar $1^{+/+}$males, but none of the changes were more than twofold. There were very few changes in the female Gpbar $1^{-1-}$ mice on HFD when compared with their WT littermates (Supplementary Figure 1B and C). Livers from male Gpbar $1^{-1}$ mice on HFD showed elevated mRNA expression levels of several genes involved in cholesterol, BA, fatty acid, and glucose metabolism when compared with those from their WT littermates, but none of the changes were greater than twofold, with the exception of $A b c b 4$ and Cyp 7a1 in KO males. Female Gpbar $1^{-/-}$livers on HFD were not different from Gpbar $1^{+\uparrow+}$ controls (Supplementary Figure 2, see section on supplementary data given at the end of this article). Since Gpbar1 has also been shown to be active in macrophages (Kawamata et al. 2003, Keitel et al. 2007, 2008b), we investigated the cytokine levels in the livers from 

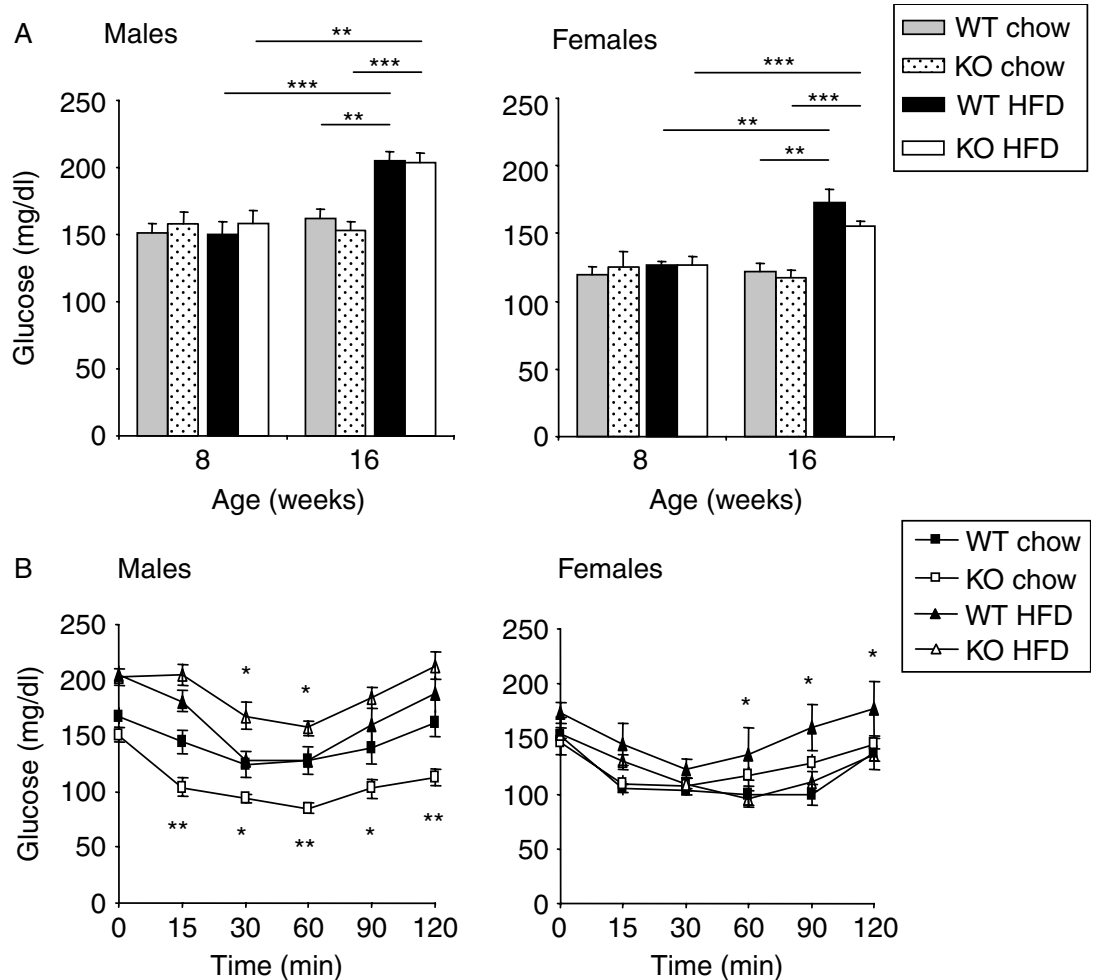

Figure 4 (A) Fasting blood glucose levels and (B) blood glucose levels after ITT of Gpbar1 and Gpbar1 $1^{+/+}$mice on chow and HFD. Individual values were measured from 11 to 17 mice per group on chow and HFD. Error bars represent \pm S.E.M. ${ }^{*} P<0 \cdot 05,{ }^{* *} P<0 \cdot 01$, and $* * * P<0 \cdot 001$.

Gpbar1 $^{-/-}$and Gpbar1 ${ }^{+/+}$mice on HFD. There was no statistically significant difference in the following cytokines: Il $1 a, I l 1 b, I l 15, I l 16$, Il18, and Ifna. Similarly, there were no differences in the BAT and WAT cytokine profiles between Gpbar1 $1^{-/-}$and Gpbar1 ${ }^{+/+}$mice on HFD (data not shown).

\section{Discussion}

BAs have been implicated in the regulation of energy homeostasis and glucose metabolism (Houten et al. 2006, Keitel et al. 2008b, Nguyen \& Bouscarel 2008). It has been shown recently that BAs inhibit diet-induced obesity and prevent the development of insulin resistance (Watanabe et al. 2006, Kobayashi et al. 2007). Gpbar1 (TGR5/M-Bar) is the only known membrane-bound receptor for BAs, expressed at very high levels in the epithelial cells of the gallbladder, as well as in the enteroendocrine cell lines NCI-H716 and STC-1 (Maruyama et al. 2002), and in the liver sinusoidal endothelial and Kupffer cells (Keitel et al. 2007, 2008a). It has been shown that the activation of GPBAR 1 by BAs induces the secretion of GLP-1 (Katsuma et al. 2005). The administration of the natural GPBAR1 agonist, oleanolic acid, to diet-induced obese mice has led to decreased blood glucose and insulin levels (Sato et al. 2007). The activation of GPBAR 1 by a potent and selective agonist, CA-derived INT-777 (Thomas et al. 2009), in diet-induced obese mice has resulted in increased resistance to $\mathrm{BW}$ gain and hepatic steatosis, and improved maintenance of glucose homeostasis and insulin sensitivity.
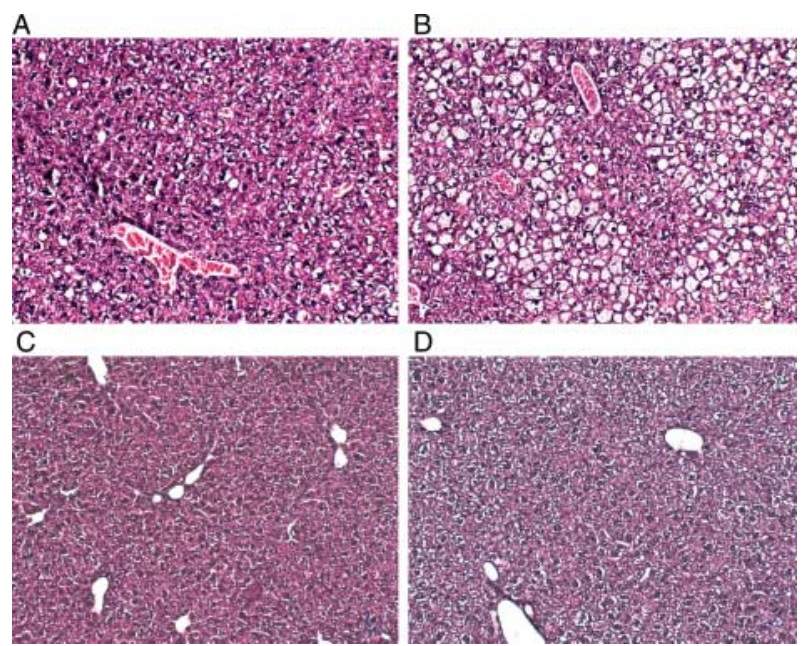

Figure 5 Histological analysis of livers from (A) Gpbar1 ${ }^{+/+}$males on HFD, (B) Gpbar1 ${ }^{-1-}$ males on HFD, (C) Gpbar1 ${ }^{+/+}$females on HFD, and (D) Gpbar1 ${ }^{-1-}$ females on HFD. Three mice per group were analyzed. Magnification $\times 100$. 
To investigate the physiological role of GPBAR 1 in vivo, we placed Gpbar1 ${ }^{-/}$males and females together with Gpbar $1^{+/+}$littermates on chow and HFD for 8 weeks. Our analysis suggests a gender-dependent regulation of the GPBAR1 function. There were no significant differences in the BWs between the corresponding Gpbar1 ${ }^{-/-}$and Gpbar $1^{+/+}$groups of mice on chow and HFD (Fig. 1). When independently generated Gpbar1 KO mice (Maruyama et al. 2006) were challenged with HFD, increased BW in female Gpbar1 $1^{-1-}$ mice was observed. One possibility for the discrepancy between the two results is the different background strain of mice, 129/sv $\times$ C57BL/ 6 backcrossed four times into C57BL/6N for Maruyama et al. (2006), and C57BL/6J for the Gpbar1 KO line described here.

There were no significant differences in the hepatic lipid composition between the corresponding Gpbar1 ${ }^{-/}$and Gpbar $1^{+/+}$groups of mice on chow and HFD, with the exception of lower FC in Gpbar1 $1^{-1-}$ males on HFD and higher FC in Gpbar1 ${ }^{-/-}$females on chow, when compared with their WT littermates (Fig. 2). However, Gpbar1 ${ }^{-/-}$ females on both chow and HFD had lower plasma total cholesterol levels, as well as Chol-VLDL, Chol-LDL, and Chol-HDL, than their WT littermates. In the Gpbar1-1males, only the total cholesterol and Chol-LDL levels were lower than those in the Gpbar1 $1^{+/+}$littermates (Fig. 3A). Female Gpbar1 ${ }^{-/-}$mice on chow had lower plasma total TG, TG-VLDL, TG-LDL, and TG-HDL levels than Gpbar1 ${ }^{+/+}$ females on chow, but only TG-VLDL levels were lower when on HFD. Male Gpbar1 $1^{-1-}$ mice had lower total TG and TG-LDL levels than their WT littermates when fed chow, but no differences were observed when fed HFD (Fig. 3B).

There was no difference in the fasting blood glucose levels between male and female Gpbar1 $1^{-1-}$ mice and their WT littermates on chow and on HFD at the beginning and at the end of the study (Fig. 4A). However, the ITT performed at the end of the HFD feeding revealed improved insulin sensitivity in Gpbar1 $1^{-/-}$males on chow, but impaired insulin sensitivity in Gpbar1 $1^{-/-}$males on HFD at 30 and $60 \mathrm{~min}$ after insulin administration, when compared with their WT littermates. In contrast, the female Gpbar $1^{-/-}$mice fed HFD showed improved insulin sensitivity at 60, 90, and $120 \mathrm{~min}$ after insulin administration (Fig. 4B). These results are in agreement with the analysis of another independently generated Gpbar1 KO line, where the authors have reported on impaired glucose tolerance of $\mathrm{Gpbar}^{-/-}$males, and improved glucose tolerance of transgenic mice overexpressing Gpbar1/TGR5, after 8 weeks on HFD (Thomas et al. 2009). Currently, the molecular mechanism behind the sex difference in the response to ITT is not clear. However, one can speculate that the fact that Gpbar $1^{-/-}$males on HFD are slightly heavier than their WT littermate controls may be a contributing factor to the impaired insulin sensitivity. The improved insulin sensitivity of the Gpbar1 $1^{-1-}$ males on chow and of the Gpbar1 $1^{-1-}$ females on HFD needs further investigation.
At the end of the HFD study, both male and female Gpbar1 $1^{-1-}$ mice displayed liver steatosis. But Gpbar1 $1^{-/-}$ males showed a higher degree of liver lipidosis when compared with their WT littermates or the Gpbar1 $1^{-1-}$ females on HFD (Fig. 5). These results are in agreement with recent reports that treatment of diet-induced obese C57BL/ 6J mice with the GPBAR1/TGR5-selective agonist, INT-777, led to reduction of liver steatosis (Thomas et al. 2009). Expression profile of 11 genes involved in glucose homeostasis in pancreatic islets (Supplementary Figure 1A) and of 43 genes involved in the BA, cholesterol, fatty acid, and glucose homeostasis in the BAT, WAT, and livers of Gpbar1 $1^{-1-}$ and Gpbar1 $1^{+/+}$mice on HFD did not reveal more than $2 \cdot 5$-fold changes between $\mathrm{KO}$ and WT control mice (Supplementary Figures 1B, C and 2).

Sexual dimorphism in HFD-induced obesity and metabolic alterations, and hepatic transport of taurocholate have been reported in mice and rats (Simon et al. 1999, Nishikawa et al. 2007, Hwang et al. 2010, Grove et al. 2010). In humans, key characteristics of obesity such as pattern of fat accumulation, energy homeostasis, and sensitivity of the brain to adiposity hormones, insulin and leptin, have been shown to be influenced by gender (Woods et al. 2003, Regitz-Zagrosek et al. 2007). It has been reported that male C57BL/6J mice on HFD gain more BW than the females, although the underlying mechanism of this sex differences remains unclear (Hwang et al. 2010). In this study, we used Gpbar1 KO mice on C57BL/6J genetic background and compared them to their WT littermates. In agreement with the published data, we observed that the males on HFD gained more weight than the females on HFD. But the fact that male Gpbar1 $1^{-/-}$mice responded to HFD challenge differently than Gpbar1 $1^{-/-}$females when compared with their WT littermates points to a gender-dependent regulation of Gpbar1 function. At this point, it is unclear why male and female Gpbar1 $1^{-1-}$ respond differently to HFD challenge. It is possible that the $\mathrm{KO}$ mice on HFD undergo sex-related hormonal changes that may contribute to the observed differences. It is important to note that GPBAR $1 \mathrm{KO}$ is a genetic deletion, and developmental effects may play a role.

In conclusion, our study extends the analysis of Gpbar1 $\mathrm{KO}$ mice on HFD and demonstrates that the deletion of GPBAR1 has differential effect in males and females. Recently, GPBAR 1 has emerged as a promising new target in metabolic disease. Although the relevance of our data to human GPBAR1 biology is not yet established, our findings of a sexual dimorphism in GPBAR1 function may have important implications for designing of GPBAR1-based therapeutic strategies.

\section{Supplementary data}

This is linked to the online version of the paper at http://dx.doi.org/10.1677/ JOE-10-0009. 


\section{Declaration of interest}

All authors are employed by Merck Research Laboratories.

\section{Funding}

The work is funded by Merck Research Laboratories.

\section{Acknowledgements}

We thank M DeLorenzo for excellent technical assistance and Dr M Bayne for helpful discussions and advice.

\section{References}

Allain CC, Poon LS, Chan CS, Richmond W \& Fu PC 1974 Enzymatic determination of total serum cholesterol. Clinical Chemistry 20 470-475

Burrier RE, Smith AA, McGregor DG, Hoos LM, Zilli DL \& Davis HR Jr 1995 The effect of acyl CoA: cholesterol acyltransferase inhibition on the uptake, esterification and secretion of cholesterol by the hamster small intestine. Journal of Pharmacology and Experimental Therapeutics 272 156-163.

Fiorucci S, Mencarelli A, Palladino G \& Cipriani S 2009 Bile-acid-activated receptors: targeting TGR 5 and farnesoid-X-receptor in lipid and glucose disorders. Trends in Pharmacological Sciences 17 139-159.

Folch J, Lees M \& Sloane Stanley GH 1957 A simple method for the isolation and purification of total lipides from animal tissues. Journal of Biological Chemistry 226 497-509.

Foord SM, Bonner TI, Neubig RR, Rosser EM, Pin JP, Davenport AP, Spedding M \& Harmar AJ 2005 International Union of Pharmacology. XLVI. G protein-coupled receptor list. Pharmacological Reviews 57 279-288.

Grove KL, Fried SK, Greenberg AS, Xiao XQ \& Clegg DJ 2010 A microarray analysis of sexual dimorphism of adipose tissues in high-fat-diet-induced obese mice. International Journal of Obesity DOI:10.1038/ijo.2010.12.

Hoffler U, Hobbie K, Wilson R, Bai R, Rahman A, Malarkey D, Travlos G \& Ghanayem BI 2009 Diet-induced obesity is associated with hyperleptinemia, hyperinsulinemia, hepatic steatosis, and glomerulopathy in C57Bl/6J mice. Endocrine 36 311-325.

Houten SM, Watanabe M \& Auwerx J 2006 Endocrine functions of bile acids. EMBO Journal 25 1419-1425.

Hwang LL, Wang CH, Li TL, Chang SD, Lin LC, Chen CP, Chen CT, Liang KC, Ho IK, Yang WS et al. 2010 Sex differences in high-fat diet-induced obesity, metabolic alterations and learning, and synaptic plasticity deficits in mice. Obesity 18 463-469.

Ikemoto S, Takahashi M, Tsunoda N, Maruyama K, Itakura H, Kawanaka K, Tabata I, Higuchi M, Tange T, Yamamoto TT et al. 1997 Cholate inhibits high-fat diet-induced hyperglycemia and obesity with acyl-CoA synthetase mRNA decrease. American Journal of Physiology 273 E37-E45.

Katsuma S, Hirasawa A \& Tsujimoto G 2005 Bile acids promote glucagon-like peptide-1 secretion through TGR 5 in a murine enteroendocrine cell line STC-1. Biochemical and Biophysical Research Communications 329 386-390.

Kawamata Y, Fujii R, Hosoya M, Harada M, Yoshida H, Miwa M, Fukusumi S, Habata Y, Itoh T, Shintani Y et al. 2003 A G protein-coupled receptor responsive to bile acids. Journal of Biological Chemistry $2789435-9440$.

Keitel V, Reinehr R, Gatsios P, Rupprecht C, Gorg B, Selbach O, Haussinger D \& Kubitz R 2007 The G-protein coupled bile salt receptor TGR 5 is expressed in liver sinusoidal endothelial cells. Hepatology 45 695-704.

Keitel V, Donner M, Winandy S, Kubitz R \& Haussinger D 2008a Expression and function of the bile acid receptor TGR 5 in Kupffer cells. Biochemical and Biophysical Research Communications 372 78-84.

Keitel V, Kubitz R \& Haussinger D $2008 b$ Endocrine and paracrine role of bile acids. World Journal of Gastroenterology 14 5620-5629.
Keitel V, Cupisti K, Ullmer C, Knoefel WT, Kubitz R \& Haussinger D 2009 The membrane-bound bile acid receptor TGR 5 is localized in the epithelium of human gallbladders. Hepatology 50 861-870.

Kobayashi M, Ikegami H, Fujisawa T, Nojima K, Kawabata Y, Noso S, Babaya N, Itoi-Babaya M, Yamaji K, Hiromine Y et al. 2007

Prevention and treatment of obesity, insulin resistance, and diabetes by bile acid-binding resin. Diabetes 56 239-247.

Li C, Najafi H, Daikhin Y, Nissim IB, Collins HW, Yudkoff M, Matschinsky FM \& Stanley CA 2003 Regulation of leucine-stimulated insulin secretion and glutamine metabolism in isolated rat islets. Journal of Biological Chemistry 278 2853-2858.

Maruyama T, Miyamoto Y, Nakamura T, Tamai Y, Okada H, Sugiyama E, Nakamura T, Itadani H \& Tanaka K 2002 Identification of membrane-type receptor for bile acids (M-BAR). Biochemical and Biophysical Research Communications 298 714-719.

Maruyama T, Tanaka K, Suzuki J, Miyoshi H, Harada N, Nakamura T, Miyamoto Y, Kanatani A \& Tamai Y 2006 Targeted disruption of G protein-coupled bile acid receptor 1 (Gpbar1/M-Bar) in mice. Journal of Endocrinology 191 197-205.

Nguyen A \& Bouscarel B 2008 Bile acids and signal transduction: role in glucose homeostasis. Cellular Signalling 20 2180-2197.

Nishikawa S, Yasoshima A, Doi K, Nakayama H \& Uetsuka K 2007 Involvement of sex, strain and age factors in high fat diet-induced obesity in C57BL/6J and BALB/cA mice. Experimental Animals 56 263-272.

Regitz-Zagrosek V, Lehmkuhl E \& Mahmoodzadeh S 2007 Gender aspects of the role of the metabolic syndrome as a risk factor for cardiovascular disease. Gender Medicine 4 S162-S177.

Salisbury BG, Davis HR, Burrier RE, Burnett DA, Bowkow G, Caplen MA, Clemmons AL, Compton DS, Hoos LM, McGregor DG et al. 1995 Hypocholesterolemic activity of a novel inhibitor of cholesterol absorption, SCH 48461. Atherosclerosis 115 45-63.

Sato H, Genet C, Strehle A, Thomas C, Lobstein A, Wagner A, Mioskowski C, Auwerx J \& Saladin R 2007 Anti-hyperglycemic activity of a TGR5 agonist isolated from Olea europaea. Biochemical and Biophysical Research Communications 362 793-798.

Simon FR, Fortune J, Iwahashi M, Bowman S, Wolkoff A \& Sutherland E 1999 Characterization of the mechanisms involved in the gender differences in hepatic taurocholate uptake. American Journal of Physiology 276 G556-G565.

Thomas C, Auwerx J \& Schoonjans K 2008a Bile acids and the membrane bile acid receptor TGR 5 - connecting nutrition and metabolism. Thyroid 18 167-174.

Thomas C, Pellicciari R, Pruzanski M, Auwerx J \& Schoonjans K $2008 b$ Targeting bile-acid signalling for metabolic diseases. Nature Reviews. Drug Discovery 7 678-693.

Thomas C, Gioiello A, Noriega L, Strehle A, Oury J, Rizzo G, Macchiarulo A, Yamamoto H, Mataki C, Pruzanski M et al. 2009 TGR5-mediated bile acid sensing controls glucose homeostasis. Cell Metabolism 10 167-177.

Vassileva G, Golovko A, Markowitz L, Abbondanzo SJ, Zeng M, Yang S, Hoos L, Tetzloff G, Levitan D, Murgolo NJ et al. 2006 Targeted deletion of Gpbar1 protects mice from cholesterol gallstone formation. Biochemical Journal 398 423-430.

Watanabe M, Houten SM, Mataki C, Christoffolete MA, Kim BW, Sato H, Messaddeq N, Harney JW, Ezaki O, Kodama Tet al. 2006 Bile acids induce energy expenditure by promoting intracellular thyroid hormone activation. Nature 439 484-489.

Wei J, Qiu de K \& Ma X 2009 Bile acids and insulin resistance: implications for treating nonalcoholic fatty liver disease. Journal of Digestive Diseases $\mathbf{1 0}$ 85-90.

Woods SC, Gotoh K \& Clegg DJ 2003 Gender differences in the control of energy homeostasis. Experimental Biology and Medicine 228 1175-1180.

Received in final form 9 March 2010

Accepted 30 March 2010

Made available online as an Accepted Preprint 30 March 2010 